Bibliothecae mutantur - QUARe, QUEMAdMOdUm ET QUID ATTINET?

Transformations de la composition thématique des bibliothèques du Royaume de Hongrie aux XVe-XVI ${ }^{e}$ siècles

\title{
István Monok
}

L'ordre des bibliothèques, la composition thématique du corpus qu'elles conservent subit des transformations permanentes. La quantité des livres y conservés est tantôt en croissance (acquisitions), tantôt en décroissance (pertes, vols, destructions ou amoindrissement volontaire du corpus). L'ordre thématique, l'ordo librorum est également sujet au changement, en partie parce que la composition thématique des sciences et des connaissances générales est en mouvement permanent, en partie parce que les autorités qui entretiennent les bibliothèques réservent à ces institutions des fonctions toujours différentes. Les changements qui affectent l'ordo librorum sont donc toujours en même temps des changements thématiques. Souvent, ceux aui viennent de prendre en charge les collections, réarrangent les livres, pour faire passer un nouveau message qu'exprime leur ordre. Au sein de certaines communautés culturelles il existe certains phénomènes généraux se manifestant dans le milieu des bibliothèques qui sont absulement inconnus dans d'autres communautés. (Ces comparaisons ont leur raison d'être actuellement, au début de ce XXI siècle. Je dirais même qu'elles s'imposent. Si l'Europe, chrétienne, occidentale, veut se définir une identité (qui ne s'épuise pas dans la liberté du commerce et des investissements), elle doit impérativement connaître ceux et celles qui arrivent sur notre continent. Il s'agit de gens avec des convictions fondamentalement différentes des nôtres. Or, les convictions différentes incriminées peuvent éventuellement s'éclairer par leurs idées sur les bibliothèques (en supposant qu'ils ont déjá entendu parler de livres et de bibliothèques).

Dans la période étudiée par notre colloque, les exemples provenant de l'Europe occidentale sont extrêmement variés. Il existe un tas de 
définitions du substantif 'Europe' et de l'adjectif 'européen'. Quelque historiens hongrois ont souligné que le continent européen avait vu la formation de divers types de feudalisme, ce qui a conduit á la naissance de trois grandes régions européennes historiques, notamment l'occidentale, l'orientale et la centrale (Zwischen-Europa). Du pont de vue de l'histoire du livre, ces trois régions sont très éloignées les unes des autres. L'Europe orientale appartient au monde de l'orthodoxie orientale, or, dans cette région du monde le livre et les bibliothèques jouent, dans la transmission des traditions culturelles, un rôle différent que plus à l'ouest. Les extrémités occidentales et méridionales de l'Europe occidentale ressemblent à la région centrale par le quantité des livres et les possibilités de leur acquisition. On peut penser aux diverses théories centre-périphérie et il n'est pas interdit non plus d'évoquer la thèse de Frédéric Barbier, qui considère l'axe Anvers-Venise, se prolongeant au cours du XVIII siècle jusquá Londres, comme le centre européen de la production de livres. Certes, la transformation du contenu des bibliothèques se déroule selon des coordonnés fondamentalement différents à Paris, à Breslau, à Cassovie ou à Sárospatak. La culture des territoires comme la région parisienne est émettrice, tandis que les autres villes ici énumérées appartiennent à des régions réceptrices. Les territoires intermédiaires, quant á eux, jouent le rôle de transmetteur ou de filtre.

Quant aux mutations qu'on peut observer dans la composition du corpus des bibliothèques, le rôle des facteurs externes - non culturels, ni scientifiques ou culturels - avait été déterminant au cours des XVI-XVIII ${ }^{e}$ siècles. Le réseau institutionnel ecclésiastique, culturel et scientifique qui fonctionne très efficacement en Europe occidentale n'est pas inexistant en Europe centrale non plus, mais son développement fut empêché, il fut même carrément détruit à plusieurs reprises. Les royaumes qui avaient, malgré eux, endossé le rôle pénible de freiner les ambitions occidentales des puissances russe, cosaque, tartare et ottomane, ne disposaient que d'un seul choix pour faire fonctionner un réseau de bibliothèques: il leur fallait accepter, obtenir et conserver le plus de livres possible. Or, c'est le toujours le donateur (et jamais 
le donataire) qui détermine la nature et le contenu du corpus reçu 'en cadeau'.

Dans ma présente étude, je me permets d'évoquer un certain nombre de cas ou les milieux intellectuels de Hongrie et de Transylvanie, en poursuivant une politique consciente d'orientation du contenu de leurs bibliothèques, ont réussi à suivre de près les courant intellectuels à la mode en Europe occidentale. J'évoquerai aussi quelques exemples de réceptions tardives, voire carrément archaiques. Bien entendu, je ne manquerai pas de traiter les causes profondes de ces phénomènes.

La commémoration du 500 anniversaire de la Réforme fut l'occasion, en plusieurs pays européens, de la mise en place de quelques programmes culturels très ambitieux. Un tas de colloques internationaux fut par exemple consacré à la problématique de la devotio moderna, aux origines intellectuelles extrêmement variées de la Réforme protestante et au processus de la formation des églises protestantes, organisées chacune autour des principes et des intérêts communs. La vie des communautés fidèles à Rome a également subi quelques transformations, dont l'esprit a été déterminé au concile de Trente. Les colloques en question ont illustré cette mutation par l'analyse des transformations du contenu des livres publiés et par la mise en valeur du rôle importantissime de l'imprimerie du point de vue de l'histoire de la communication. Je tiens à souligner que les transformations qu'on peut observer dans la composition thématique des bibliothèques constituent également un indice digne de notre attention.

Cela demeure vrai même si l'on doit admettre que nous connaissons très peu d'institutions ou de familles dont les bibliothèques seraient caractérisés par un développement organique. L’analyse du caractère des transformations doit passer par leur comparaison avec les sources provenant des institutions et des groupes de personnes privées étudiés. Cela me paraît faisable même dans les régions centrales du continent européen. De la période d'avant le milieu du XVI e siècle, il subsiste un nombre assez limité de recensements de livres produits en Royaume de Hongrie. Cela remonte prioritairement à des raisons d'ordre politique - 
les guerres avec les Ottomanes, la double élection royale (Ferdinand I ${ }^{\text {er }}$ Habsbourg et János Szapolyai) après la défaite de Mohács -, pourtant, il convient aussi de noter que la carence en papier et parchemin explique également la lenteur des progrès de l'imprimerie dans la région. Certes, les matériaux des périodes précédentes furent, partout en Europe, réutilisés pour écrire dessus, mais surtout pour relier des livres; dans les régions centrales, on se servit de ce procédé plus souvent qu'ailleurs.

Comparons, pour commencer, la bibliothèque du chapitre cathédral d'Angers (proche des régions centrales) á celles, contemporaines, situées en Royaume de Hongrie. L'on verra un évident écart quantitatif, mais quant au contenu, les différences ne sont pas énormes. A Angers, on trouve, en 1467/1472, 309 volumes'; à Presbourg, en 1425, 83 volumes²; à Veszprém, en 1435, 153 volumes ${ }^{3}$; enfin, à Zagreb, en 1433, 231. ${ }^{4}$ L'on ne peut pas suivre de près les transformations en contenu des ces dernières bibliothèques, par contre, celle de la confraternitas des 24

$1 \quad$ Jean-Michel MATz, La bibliothèque du chapitre cathédral d'Angers d'après l'inventaire de 1472, = Formation intellectuelle et culture du clergé dans les territoires angevins (milieu du XIII - fin du XVe siècle), sous la dir. de Marie-Madeleine de Cevins, Jean-Michel MATZ, Rome, 2005 (Collection de l'École française de Rome, 349), 185-220.

2 Csapodi Csaba-Csapodine Gardonyi Klára, Bibliotheca Hungarica, Kódexek és nyomtatott könyvek Magyarországon 1526 elött, vol. I-IV, Budapest, MTAK, 1988-1995 (A Magyar Tudományos Akadémia Könyvtárának Közleményei - Publicationes Bibliothecae Academiae Scientiarum Hungaricae, 23, 31, 33, 34.), III, 1994, 114-117: Nr. 904-943. Sur la culture du livre á Presbourg, voir: Juraj ŠEDivý, Mittelalterliche Schriftkultur im Pressburger Kollegiatkapitel, Bratislava, Verlag Chronos, 2007.

3 Csapodi-GÁrdonyi, Bibliotheca Hungarica, III, 1994, 122-130: Nr. 977-1100.; László Solymosi, The library of Veszprém cathedral and ist borrowers in the late Middle Ages, = Infima aetas Pannonica. Studies in Late Medieval Hungarian History. ed. by Péter E. Kovacs, Kornél Szovak. Budapest, Corvina, 2009, 260-272.

4 CSApodi-GÁRdonyi, Bibliotheca Hungarica, III, 1994, 130-134: Nr. 1106-1167; pour l'analyse du corpus, voir: Előd NemerkenYi, Cathedral Libraries in Medieval Hungary, Library History, vol. 20. March 2004, 7-17.; Edit MADAs, Les bibliothèque des chapitres de Veszprém, de Presbourg et de Zagreb d'après leurs inventaires, = CenvinsMatz, sous la dir. de, Formation intellectuels... 2005, 221-230. 
paroisses de région de Zips - on l'appelle souvent la bibliothèque médiévale de Löcse - nous est connue dans les détails, ce qui nous permet de faire des observations générales par voie d'analogie. Sur le fonctionnement de la confraternitas entre 1248 et 1674 , nous avons d'amples renseignements ${ }^{5}$, quant à la bibliothèque, son existence est attestée depuis le début du $\mathrm{XV}^{e}$ siècle $^{6}$ par le document intitulé Elenchus librorum (administré sans interruption) jusquá la fin du XVI e siècle, lorsque la bibliothèque a cessé d'acquérir de nouveaux livres. Des 412 objets mentionnés 158 sont des manuscrits, 254 sont des imprimés (dont 76 incunables, le reste - 174 objets - date du XVI e siècle). Il est très regrettable que l'Elenchus ne précise pas le titre des ouvrages luthériens. Pour toute indication, on trouve „Habentur et libri Lutheranici 52". Au-dessus de la chapelle Saint-Georges, faisant partie de l'église Saint-Jacques, Johann Henckel (1481-1539) a créé la collection connue aujourd'hui sous le nom de bibliothèque médiévale de Löcse: il s'agit de la réunion des bibliothèques paroissiales médiévales de la région de Zips, complétées de livres provenant d'autres établissements ecclisastiques locaux. Cette bibliothèque était restée á sa place jusquá la fin du XVIII siècle: en 1790, Ignace Batthyány, évêque de Transylvanie (1741-1798), l'a achetée pour la transporter à Gyulafehérvár8. Dans la bibliothèque Batthyány à Gyulafehérvár, l'on peut toujours étudier les not

5 Florian HoLıк, Die erste gelehrte Gesellschaft in Ungarn, Ungarische Jahrbücher, 2(1923) (Berlin-Leipzig), 383-399.

6 András Vizkelety, Die Fraternitas XXIV plebanorum civitatum regalium in Oberungarn und der Handschriftenbestand Zipser Pfarreibibliotheken, = Pfarreien im Mittelalter. Deutschland, Polen, Tschechien und Ungarn im Vergleich, Vom 30. November bis 2. Dezember 2006 am Max-Planck-Institut für Geschichte eine Tagung zum Thema Pfarreien in Mitteleuropa im Mittelalter, Hrsg. von Nathalie Kruppa, Göttingen, Vandenhoeck und Ruprecht, 2008, 327-338. Eva Selecká Mârza, A Középkori Löcsei Könyvtár, Szeged, Scriptum, 1997 (Olvasmánytörténeti dolgozatok, VII.) Cf. Gabriela Žırisitová, Schweizerische Bücher in den ältesten Bibliotheken von Leutschau und Bartfeld. Buch und die Privatbibliotheken der Stadtbürger in der frühen Neuzeit, = Orbis Helveticorum. Das Schweizer Buch und seine mitteleuropäische Welt, hrsg. von Viliam Čı̌̌AJ, JanAndrea Bernhard, Bratislava, HuSAV, 2011, 237-249.

8 JAKó Zsigmond, Batthyány Ignác a tudós és a tudományszervező, Erdélyi Múzeum, LIII(1991), 76-99. 
es manuscrites sur les pages des incunables de la collection. ${ }^{9}$ Il ressort de leur examen que 76 volumes (91 ouvrages) se trouvaient déjá à Lőcse au $\mathrm{XV}^{\mathrm{e}}$ siècle. Le nombre des livres imprimés á telle ou telle ville européenne est proportionnelle à la distance qui en sépare Lőcse: Nuremberg, Augsburg, Ulm, Cologne, Venise, Milan, Bâle, Rome, Lyon. Il est étonnant de voir la présence d'un seul incunable produit à Cracovie, ville pourtant relativement proche. La composition thématique de la collection correspond aux exigences des utilisateurs présumés: premières éditions des ouvrages des Pères de l'Église, collections de sermons, explications de la Bible, épîtres des papes. L'étude des sources permet la description de deux phenomènes. Le premier est l'exemple de Johann Henckel (1486-1539), l'autre, l'exploitation du corpus médiéval dans les villes devenues protestantes. Johann Henckel - ayant étudié la théologie, les lettres et la jurisprudence entre 1506 et 1510 à Cracovie, à Vienne, à Bologne, et à Padoue - a été nommé curé paroissial à Lőcse. Il prenait grand soin de la collection de livres qu'il avait réunies. ${ }^{10}$ Une partie importante de ses livres est restée à Lőse après son départ à Kassa. En 1520, il a rejoint la cour royale en tant que confesseur de la reine Marie de Habsbourg. Ami de Miklós Oláh (historien, futur évêque d'Esztergom), correspondant régulier d'Erasme, il entretenait des rapports avec Martin Luther et Philipp Melanchthon également. A la fin de sa vie, il se convertit à la confession luthérienne. Sa bibliothèque - dont les pièces sont plus tard entrées dans une collection urbaine à usage commun, à Lőcse - est d'orientation humaniste: éditions récentes des Pères de l'Eglise, traités des humanistes contemporains, imprimés luthériens. Au cours du XVI ${ }^{\mathrm{e}}$ siècle, la ville a enrichi cette collection

9 Catalogus incunabulorum Bibliothecae Batthyányanae, conscriptus Petro Kulcsár, Szeged, JATE, 1965; Selecká Mârza, A Középkori Lö́csei Könyvtár, 1997.

10 Une synthèse sur la vie et les études de Henckel: Jakó Zsigmond, Várad helye középkori egyháztörténetünkben, = JAKó Zsigmond, Írás, könyv, értelmiség, Tanulmányok Erdély történelméhez, Bukarest, Kriterion, 1976, 138-169. Voir aussi KRIstóF Ilona, Egyházi középréteg a késő középkori Váradon (1440-1526), Pécs, Pécsi Történettudományért Egyesület, 2014 (Thesaurus historiae ecclesiasticae in Universitate Quiqueecclesiensi, 4), 145-146, 289-298. 
d'origine médiévale avec quelques ouvrages luthériens. Les livres provenant des donations privées renforcent également l'orientation spiritualiste, devotio moderna et protestante de la collection. 27 des livres de Georg Molner, humaniste de la région de Zips, 10 des livres de Caspar Polirer, curé de Lőcse subsistent jusquà aujourd'hui: parmi les premiers, on trouve un tas d'auteurs de Wittenberg."

La transformation thématique ici présentée des bibliothèques est une mutation organique, qui suit certes lentement, mais jamais avec un trop grand retard, les chagements des courants intellectuels. Les collections privées et les donations qui entrent des les bibliothèques institutionnelles illustrent également cette tendance.

L'exemple de Bártfa est semblable. En suivant de près l'histoire de la bibliothèque de l'église Saint-Gilles, l'on voit qu'au temps médiévaux la collection se limitait à quelques livres cérémoniaux (1435/1437,1451/1455, 1466, 1495). ${ }^{12}$ Dans la suite, les 83 volumes ayant appartenu aux legs de Georgius Petri (1509) 13 sont majoritairement constitués d'éditions imprimées d'auteurs classiques, de plusieurs pièces de la série bâloise des Pères d'Eglise latins (par Johann Amerbach), ainsi que de maints ouvrages de grammatica, de arte carmina, de poetica d'auteurs humanistes contemporains. Comme il va de soi, on trouve de la théologie médiévale et quelques vitae patrum sur le registre des livres. C'est une bibliothèque relativement moderne, qui a probablement contribué au rayonnement de la littérature humaniste du début du XVI ${ }^{\mathrm{e}}$ siècle. Par contre, les 16 volumes que Balthasar Blutfogel offrit à la bibliothèque aux alentours de 1530 n'a nullement renforcé le caractère moderne de la collection: il s'agit d'ouvrages des Pères d'Eglise et des traités théologiques, imprimés

11 Selecká MÂrza, A Középkori Löcsei Könyvtár, 1997, 41.

1221 volumes: Csapodi-GÁrdonyi, Bibliotheca Hungarica, III, 1994, 140-142: Nr. 1203-1224.

13 ÁвеL Jenő, A bártfai Sz.-Egyed temploma könyvtárának története, Budapest, MTA, 1885, 62-77.; CsApodi-GÁRdonyi, Bibliotheca Hungarica, III, 1994, 256-261: Nr. 2351-2434. 
dans la dernière décennie du XVI ${ }^{\mathrm{e}}$ siècle. ${ }^{14}$ Selon un registre daté de 1640, les livres d'un des professeurs de l'école locale, un certain Gaspar Seiffrid, venaient d'entrer à la bibliothèque de la ville. ${ }^{15}$ L'inscription sur le registre en question dit: „Anno 1640 Registrum librorum domini Gaspari Seffridii olim Rectoris Scholae nostrae industrii" (141 livres) - ce passage latin permet plusieurs interprétations, puisque nous ne savons rien de la vie de Seiffrid. Á quelle période était-il recteur de l'école? Jadis (olim). Le recensement a-t-il été effectué après sa démission, après sa mort ou plus tard? Ou, éventuellement, beaucoup plus tard? La plupart des livres qui passerent à la collection de la ville datent essentiellement du XVI ${ }^{\mathrm{e}}$ siècle: il s'agit d'un corpus au moins aussi intéressant que celui qu'Imre Forgács a accordé en 1588 à l'école de Trencsén ${ }^{16}$, ou bien la bibliothèque de Stanislas Thurzó, conservée dans sa maison d'Augsburg. ${ }^{17}$ Le corpus Seiffrid réunit les éditions humanistes des auteurs antiques et des Pères de l'Église (surtout les traductions latines des Pères grecs, puis les oeuvres complètes d'Augustinus), ainsi que des éditions scolaires des grands textes de la tradition et quelques ouvrages inspirés par la Réforme wittenbergoise. On peut donc dire que l'élargissement thématique de la bibliothèque de Bártfa s'est arrêtée á la dernière décennie du XVI e siècle. Au XVII e siècle, les catholiques ont mis la main sur la collection, récupérée en 1705 par les protestants. A cette

14 ÁвеL, A bártfai ... 1885, 78-88.

15 Á 1657, sajtó alá rend. VARGA András, munkatárs MoNoK István, utószó MoNoK István, VARGA András, Budapest-Szeged, JATE, MTAK, 1986 (Adattár XVIXVIII. századi szellemi mozgalmaink történetéhez, 13), 144-147.

16 Magyarországi magánkönyutárak, II, (1580-1721), sajtó alá rend. FARKAS Gábor, Katona Tünde, Latzkovits Miklós, Varga András. Szeged, 1992 (Adattár XVI-XVIII. századi szellemi mozgalmaink történetéhez, 13/2), 3-9.

17 A magyar könyvkultúra múltjából, Iványi Béla cikkei és anyaggyújtése, par HeRNER János, Monok István, Szeged, JATE, 1983 (Adattár XVI-XVIII. századi szellemi mozgalmaink történetéhez, 11), 489-490.; Monok István, A müvelt arisztokrata, A magyarországi fönemesség olvasmányai a XVI-XVII. században, Budapest-Eger, Kossuth, EKF, 2012 (Kulturális örökség), 160-162. 
dernière occasion, un nouveau recensement a été effectué..$^{18}$ On y trouve 84 pièces (122 ouvrages), ce qui prouve la relative négligence des auteurs du recensement, puisquá la suivante passation des pouvoirs, en 1725, les catholiques y ont trouvé 138 livres $^{19}$. Aucun nouveau livre depuis les donations du XVI e siècle et la bibliothèque de Seiffrid.

Dans ce dernier exemple la transformation thématique, le processus d'enrichissement de la bibliothèque fut interrompue par des changements survenus dans le domaine de la politique ecclésiastique. Notons un phénomène digne d'intérêt: chaque fois qu'une bibliothèque ecclésiastique se voit enrichir d'un corpus qui aurait sa place véritable dans une collection scolaire ou humaniste, cela équivaut pratiquement á sa perte, puisqu'il n'est pas utilisé.

Par contre, nous pouvons énumérer un tas d'exemples ou le corpus des collections scolaires accroît sa 'valeur d'usage' grâce aux donations et à l'élargissement thématique. Ce qui doit surtout retenir notre attention dans la suite c'est la volonté permanente de la part de l'Eglise, du conseil municipal, des professeurs et des patrons des écoles de surveiller le processus de la diffusion des connaissances. Quels sont les livres que les écoliers pourront lire? Au tournant des XVIII - XIX ${ }^{e}$ siècles, la bibliothèque des enseignants finira par être soigneusement séparée de celle des écoliers, ce qui aura pour résultat que les étudiants seniors ne pourront plus remplir le poste du responsable de la collection. Être bibliothécaire sera désormais une

18 Á Magyarországon 1526-1750, sajtó alá rend. Zvara Edina, Szeged, Scriptum, 2001 (Adattár XVI-XVIII. századi szellemi mozgalmaink történetéhez, 19/1), 195-198. Ámel, A bártfai ... 1885, 130-137.; Zvara, Adattár 19/1, 2001, 198-202. 
profession á part ${ }^{20}$ (l’un des premiers en Hongrie sera le bibliothécaire de l'école de Sárospatak, János Szombathy ${ }^{21}$ ).

L'école scolaire protestante majeure du XVI ${ }^{e}$ siècle en Hongrie fut celle de Németújvár. Grâce au soutien de son patron, la famille Batthyány, elle a fonctionné très efficacement entre 1569 et 1628. La bibliothèque a fermé ses portes en 1634, à cause de la conversion à la foi catholique du pater familias. La famille offrait régulièrement à l'école le corpus scolaire et théologique de la collection de la cour familiale. L'actualité sensationnelle de la collection ${ }^{22}$ était assurée par les exilés protestants ${ }^{23}$, arrivés de Styrie et de Carinthie. Plus tard, dans la première décennie de la guerre de trente ans, les domaines de la famille Batthyány ont également accueilli des protestants contraints de quitter la Boheme, la Bavière et le Württemberg.

La composition thématique de la bibliothèque de l'école municipale de Brassó fut élaborée par Johann Honter (1498-1549), imprimeur, curé, puis pasteur saxon. Il avait repris la majeure partie des livres ayant

20 István Monok, Qui peut-on appeler bibliothécaire du XVI au XVIII siècle en Hongrie? = Histoire des bibliothécaires, Colloque international à l'ENSSIB, Lyon, du 27 au 29 novembre 2003, sous la dir. de Dominique VARRY, Frédéric Barbier, Lyon, 2003, 23-32. livre numérique: http://www.enssib.fr/bibliothèquenumerique/documents/1337-histoire-des-bibliothecaires.pdf; МоNoK István, A hagyományos világ átváltozásai, Tanulmányok a XVIII. századi magyarországi könyvtárak történetéhez, Budapest-Eger, Kossuth, EKE (Kulturális örökség), 88.

21 Barcza József, Magyar könyvtárosok és bibliográfusok, Szombathi János (1739_ 1823), Könyvtáros, 5(1963), 32-44.

22 МоNок István, A németújvári iskola könyvtárának teológiai arculata, = Folyamatosság és változás. Egyházszervezet és hitélet a veszprémi püspökség területén a 16-17. században, szerk. KarLinszky Balázs, VARga Tibor László, Veszprém, 2018 (A veszprémi egyházmegye múltjából, 32), 269-278.

23 Mомок István, Württenbergi exulánsok Batthyány Ferenc udvarában, Magyar Könyvszemle, 119(2003), 205-21l.; István МоNoK, Die Bibliothek des Johann Jacob Knaus, Die Reste einer württenbergischen protestantischen Bibliothek in Güssing, Jahrbuch des Ungarischen Kulturinstitutes in Stuttgart, 1(2003), 138-146.; István МоNoK, Exulanten aus Bayern, Oberpfalz und Pfalz am Batthyány-Hof an der Wende des 16. und 17. Jahrhunderts, Ungarn Jahrbuch, 27(2004), 331-340. 
appartenu aux ordres religieux supprimés, puis il a enrichi la collection d'un corpus d'inspiration humaniste, composé de livres publiés au début du XVI e siècle. Il est remarquable que les donateurs postérieurs ne léguerent á l'école que des livres proprement scolaires, ce qui a contribué à 'l'archaisation' relative de la collection. A Besztercebánya, les livres utilisés à l'école furent ceux dont la bibliothèque du conseil municipal voulait se débarrasser, tandis quà Brassó, les donateurs privés ont eu recours au même procédé de sélection. Cette attitude a sérieusement affecté la variété des collections, qui ne pouvaient pas par conséquent fournir une gamme étendue de livres à leurs utilisateurs.

Un autre exemple digne d'intérêt: l'étude comparative de la bibliothèques des colleges réformés de Debrecen et de Sárospatak. Quant à Debrecen, les donations des étudiants ayant poursuivi des études à l'étranger (grâce au soutien de la ville et de l'Église réformée) correspondent aux exigences de ces mêmes autorités: il s'agit de livres conçus dans un esprit conforme à celui de la confession helvétique. Par contre, les étudiants émis par le collège de Sárospatak, patronnés par quelques familles de haute-noblesse (les Perényi, les Rákóczi) avaient la possibilité de choisir librement leur destination académique, et beaucoup d'entre eux ont opté pour des universités luthériennes ou iréniques (comme le fut par exemple Heidelberg au tournant des $\mathrm{XVI}^{\mathrm{e}}$ XVII ${ }^{e}$ siècles). De retour, ils ont offert à la bibliothèque de l' alma mater bibliotheca des livres les plus variés ${ }^{24}$. Un facteur très important dans la mutation thématique de la collection de Sárospatak fut l'intégration de la bibliothèque privée, en 1660, de la famille Rákóczi, avec tous les

24 Partiumi könyvesházak, 1621-1730, Sárospatak, Debrecen, Szatmár, Nagybánya, Zilah, sajtó alá rend. Fekete Csaba, Kulcsar György (=Bura László), Monok István, VARGA András, Budapest-Szeged, MTAK, JATE,1988 (Adattár XVIXVIII. századi szellemi mozgalmaink történetéhez 14), 7-137: Sárospatak; 139-324: Debrecen; Cf.: Monok István, Református könyvgyüjtők, református gyüjtemények a kora újkori Magyarországon, = Kálvin hagyománya, Református kulturális örökség a Duna mentén, Catalogue d'exposition, Budapesti Történeti Múzeum, 2009. október 30. - 2010. február 15. szerk. Farbaky Péter, KIss Réka, Budapest, BTM, 2009, 42-46. 
livres de la branche catholique. ${ }^{25}$ Notre dernier exemple sera celui de Nagyenyed. Une personne non identifiée, active dans la bibliothèque du collège réformé de la ville au tournant des $\mathrm{XVII}{ }^{\mathrm{e}}-\mathrm{XVIII}{ }^{\mathrm{e}}$ siècles, en a tout simplement enlevé les ouvrages des auteurs anglais, surtout, bien entendu, ceux des presbytériens. Il s'agit d'une 'purification' du corpus accessible á la jeunesse ${ }^{26}$.

Les bibliothèques institutionnelles ont vu leurcorpus considérablement élargi et leur composition thématique transformée apres l'expulsion des Ottomans du Royaume et l'intégration de la Transylvanie dans l'Empire des Habsbourgs (en tant que Grand-Duché). Les institutions de l'Église catholique, en passe de réorganisation, notamment les ordres monastiques, les séminaires responsables de la formation des clercs, les bibliothèques des chapitres et des évéchés ont bénéficié des donations souvent importantes provenant de leurs confreres occidentaux. Certes, ce ne sont pas forcément les livres les plus récents que les Occidentaux ont envoyés au bassin des Carpates, d'autant qu'à cette époque la plupart des livres nouveaux ont été déjá publiés en langue vernaculaire. Or, l'arrivée des livres quelque peu caducs, de langue latine, ont contribué à l'archaisation regrettable des lectures et de la pensée des habitants d'Europe centrale.

Jetons un coup d'oeil maintenant sur les transformations que subirent, surtout dans la période 1550-1561, les bibliothèques privées. Il nous reste relativement peu de recensement de livres qu'on pourrait qualifier de registre ${ }^{27}$ proprement dit:

25 Mомок István, A Rákóczi-család könyvtárai - Bibliotheken der Familie-Rákóczi, 1588-1660, Szeged, Scriptum, 1996 (A Kárpát-medence koraújkori könyvtárai - Bibliotheken im Karpatenbecken der frühen Neuzeit, I.)

26 Voir les notes deest dans les catalogues: Erdélyi könyvesházak II, Kolozsvár, Marosvásárhely, Nagyenyed, Szászváros, Székelyudvarhely, sajtó alá rend. MoNoK István, Németh Noémi, Tonk Sándor, Szeged, Scriptum, 1991 (Adattár XVIXVIII. századi szellemi mozgalmaink történetéhez, 16/2), 133-190. Cela exige un minimum de 5 livres différents. 
1455 Liebhard Eckenvelter, notaire de Presbourg, 40 pièces ${ }^{28}$ 1481 Georg Preirer, curé de Sopron, 14 pièces $^{29}$

1490, 1520 Ippolito d'Este (1479-1520), archévêque d'Esztergom, puis évêque d'Eger, 46 pièces ${ }^{30}$

1500 environ, Balthasar Steck, notaire de Besztercebánya, 15 pièces ${ }^{31}$

1509 Georgius Petri, curé de Bártfa, 83 pièces ${ }^{32}$

1533-1545 Clemens, bourgeois de Besztercebánya, 77 pièces, en deux recensements $^{33}$

1553 Józsa, frère dominicain, 5 pièces $^{34}$

1553 Zay Ferenc (1505-1570), noble, baronisé en 1553, 80 pièces ${ }^{35}$

1555 Rosos István, noble(?), 18 pièces $^{36}$

1558 Michael Judex de Sommelfeld (?-1558), chanoine de Presbourg, 25 pièces $^{37}$

1560 Perneszith György (?-1560), noble, intendant des domaines de la famille Nádasdy, 62 pièces $^{38}$

1561 Budai Jakab (?-1561), scribe á la cour royale, 14 pièces $^{39}$

28 CSAPODI-GÁRdonyi, Bibliotheca Hungarica, III, 1994, 152-155: Nr. 1311-1351.

29 CsApodi-GÁRdonyi, Bibliotheca Hungarica, III, 1994, 210-211: Nr. 1853-1867.

30 Csapodi-GÁrdonyi, Bibliotheca Hungarica, III, 1994, 157-159: Nr. 1364-1410.

31 Csapodi-Gárdonyi, Bibliotheca Hungarica, III, 1994, 214-215: Nr. 1898-1913.

32 Ábel, A bártfai ... 1885, 62-77.; CsApodi-GÁrdonyi, Bibliotheca Hungarica, III, 1994, 256-261: Nr. 2351-2434.

33 VArga A-Monok, Adattár 13/1, 1986, 3-6.; Magyarországi magánkönyvtárak, III, Besztercebánya, Körmöcbánya, Selmecbánya, sajtó alá rend. Vilam ČičAJ, István Monok, Noémi Viskolcz, Budapest-Szeged, OSZK, SZTE, 2003 (Adattár XVI-XVIII. századi szellemi mozgalmaink történetéhez, 13/3), 3-8. Varga A-Monok, Adattár 13/1, 1986, 8-9.

35 Varga A-Monok, Adattár 13/1, 1986, 9-11.

36 VArga A-Monok, Adattár 13/1, 1986, 11.

37 VArga A-Monok, Adattár 13/1, 1986, 11-12.

38 VArga A-Monok, Adattár 13/1, 1986, 12-14.

39 Herner-Monok, sajtó alá rend., Adattár 11, 1983, 54-55. 
En dehors de ces documents d'archives, le corpus de plusieurs bibliothèques fut reconstruit par les spécialistes ${ }^{40}$, sur la base des marques conservés dans les codex et des imprimés (notes de possessor, armoiries, dedicatio etc.). Les plus importantes de ces bibliothèques sont bien connues, comme celle de Johannes Vitéz de Zredna, ${ }^{41}$ ou la fameuse Bibliotheca Corvina. ${ }^{42}$

Je pense que l'analyse systématique de ces registres d'un point de vue thématique aboutira au résultat suivant: à partir du milieu du XV siècle, en règle générale, on rencontre dans les bibliothèques de plus en plus d'ouvrages conçus dans l'esprit de la piété vécue et personnelle. La présence des humanistes contemporains dans ces collections est de plus en plus accentuée; on peut même noter l'existence de collections exclusivement humanistes, dominées par les ouvrages d'ars poetica, d'ars historica et d'ars carmina. Je tiens aussi à souligner les ouvrages de théologie morale et de philosophie morale, ainsi que les ouvrages historiques dans lesquels les hommes d'Etat peuvent puiser des connaissances directement utilisables dans son activité politique. Les

40 Une synthèse classique et une récapitulation récente: Csaba Csapod, Ungarische Bibliotheksgeschichte, Vom Mittelalter bis zum Frieden von Szathmár (1711), Gutenberg-Jahrbuch, 49(1984), 332-357.; György Doмокоs, Letture e bibliothece nel Quattroceto in Ungheria, = L'Umanesimo Latino in Ungheria, Convegno Internazionale di Studi, 18 aprile 2005, Budapest, Istituto Italiano di Cultura, atti a cura di Adrian Papo, Gizella Nemeth Papo, Treviso, Cassamarca, 2005, 61-75.; Anna Boreczky, Book Culture in Medieval Hungary, = The Art of Medieval Hungary, ed. by Xavier Barral I Altet, Pál Lővei, Vinni Lucherini, Imre Takács, Roma, Viella, 2018 (Bibliotheca Academiae Hungariae-Roma. Studia, 7), 283-303.

41 Klára Csapodiné Gárdonyi, Die Bibliothek des Johannes Vitéz, Budapest, Akadémiai Kiadó, 1984 (Studia Humanitatis, 6.); A Star in the Raven's Shadow, János Vitéz and the Beginnings of Humanisme in Hungary, Exhibition organised by the National Széchényi Library, $14^{\text {th }}$ March $-15^{\text {th }}$ June 2008, ed. by Ferenc Földesi, Budapest, OSZK, 2008.

42 Csaba Csapodi, The Corvinian Library, History and Stock, Budapest, Akadémiai Kiadó, 1973 (Studia Humanitatis 1); Matthias Corvin, les bibliothèques princières et la genèse de l'état moderne, publié par Jean-François MaILLARD, István MoNoK, Donatella Nebbiai, Budapest, OSZK, 2009, (Supplementum Corvinianum, II.) 
textes fondamentaux de quelques professions spécifiques (jurisprudence, industrie minière) sont également accessibles dans quelques collections. A partir de 1530, l'on rencontre de plus en plus d'ouvrages protestants, mais toujours accompagnés de réponses catholiques. Les ouvrages qui rendent compte des courants intellectuels humanistes du protestantisme wittenbergois figurent plus souvent dans les collections que les textes consacrés au renouvellement et à la réorganisation de l'Église. Les registres permettent aussi de voir que les grandes éditions humanistes des maisons vénétiennes et bâloises sont des produits très recherchés par le public du Royaume de Hongrie.

Si parmi les livres de Liebhard Eckenvelter, notaire de Presbourg, on trouve déjá quelques ouvrages de jurisprudence (en latin et en allemand), ainsi que des traités philosophiques et historique, il demeure néanmoins que sa collection est toujours dominée par des sermons, des livres de prieres, des commentaires bibliques. Cinquante ans plus tard, l'autre notaire, Balthasar Steck, ${ }^{43}$ dispose déjá d'une vraie bibliothèque spécialisée. On y trouve les textes fondamentaux du droit romain et ses explications, ainsi que des traités allemands de droit urbain. Quelques grandes synthèses de théologie médiévale (Antonius Florentinus, Bartholomaeus Anglicus), ainsi que le Cato moralisans (c'est-à-dire

Dans son étude, fondée sur la publication des sources par Adalbert BAKER, Elemér MALYusz affirme que ce registre atteste l'implantation des conceptions humanistes dans la Hongrie de la fin du XVE siècle (Adalbert BAKER, Beiträge zum Geistesleben der Schemnitzer Waldbürger im XV-XVII. Jahrhundert, Karpathenland, 9(1936), 9-16, 41-49, 67-72. (Steck: p. 10.); MALYusz Elemér, Egyházi társadalom a középkori Magyarországon, Budapest, Akadémiai Kiadó, 1971, 305-377: Irodalmi élet (könyvhasználat), Sur Steck, voir: 376-377. Notons immédiatement que l'autre illustration du phénomène est le corpus de l'école de Sárospatak, voir: Meszaros István, A Szalkai-kódex és a XV. század végi sárospataki iskola, Budapest, Akadémiai Kiadó, 1972; Meszaros István, XVI. századi városi iskoláink és a „studia humanitatis”, Budapest, Akadémiai Kiadó, 1981 (Humanizmus és reformáció, 11.); Szalkai-Kódex, Szalaki László esztergomi érsek iskoláskönyve - Szalkai Codex, The Textbook of László Szalkai, Archbishop of Esztergom, facsimile, ed., and introd. Gábor SARBAK, EsztergomSárospatak, Esztergomi Főszékesegyházi Könyvtár, Sárospataki Római Katolikus Gyüjtemény, 2019. 
un Caton christianisé) complètent la collection. Le troisième clerc de la liste, Jakab de Buda, scribe à la cour royale, a été possesseur de 14 livres. Por moi, la composition thématique de sa petite collection illustre à merveille les transformations qui avaient lieu entre 1450 et 1560. Ouvrages hagiographiques, collection de sermons (franciscaine, selon toute probabilité celle de Pelbart de Temesvár), Saint Thomas d'Aquin, Bernardinus de Busti (moine franciscain), Johannes Gerson (le mathématicien), Albertus Pighius (le théologien astronome), un traité polémique contre Luther (auteur inconnu), Eobanus Hessus (le poète humaniste luthérien), enfin, le livre de Jean Calvin sur les sacrements. Piété de Moyen Âge, piété contemporaire, humanistes du XV et du $\mathrm{XVI}^{\mathrm{e}}$ siècle, ensemble avec les auteurs et textes de la Réforme - c'est ce qui me fait typique ce petite collection du milieu du XVI ${ }^{\mathrm{e}}$ siècle.

On ne sait rien de la personne de Clemens, de Besztercebánya. Il s'agit, selon toute probabilité d'un roturier, exceptionnellement érudit. Sa bibliothèque est fondée sur des opera omnia, en éditions récentes. Des ouvrages des Pères de l'Église, des humanistes contemporains, ainsi que les textes fondamentaux de la médicine (il les a offerts plus tard à l'hopital local). István Rosos (personnage également obscur) disposait, quant à lui, d'une collection typiquement humaniste, composée de bonnes éditions d'auteurs classiques et des quelques auteurs humanistes comtemporains. Perneszith György, l'intendant, partage le même goût humaniste (il peut se prévaloir de plus de livres que Rosos), mais il paraît particulièrement intéressé par les ouvrages de la première et de la deuxième génération des Réformateurs. On trouve dans sa collection quelques manifestations des grands débats théologiques et moraux du XVI ${ }^{e}$ siècle (le libre-arbitre, la présence reéelle, etc.): c'est une bibliothèque quasi-protestante, à nuance humaniste (dans l'esprit de Melanchthon et de Johann Sturm). ${ }^{44}$ Le registre des livres de Bornemisza Péter mecénásának könyvjegyzéke 1560-ból, Irodalomtörténeti Közlemények, 66(1962), 83-89.; BoRzsak István, Perneszith György könyvjegyzékéhez, Irodalomtörténeti Közlemények, 66(1962), 628-632. 
Ferenc Zay, baronisé ${ }^{45}$ atteste la présence dans sa bibliothèque de 80 livres, dont 59 sont de sujet laic. Zay s'est apparamment intéressé à la philosophie morale, à la théorie politique et à l'histoire. Quant aux livres figurant dans la rubrique libri sacri, avouons qu'ils ne sont pas tous particulièrement sacer. Certes, on y trouve des livres cérémoniaux et quelques catéchismes, mais le ton est donné par les Loci communes de Melanchthon.

Passons maintenant aux cléricaux. Georg Preirer, curé de Sopron, ne se contente pas des ouvrages dont il se sert dans son activité quotidienne. En dehors des livres cérémoniaux, des Bibles et des sermons, on trouve également des traités de droit canonique, des ouvrages de théologie très avancée, des dictionnaires, des grammaires, enfin un livre sur la vie de quelques philosophes - un aspect qu'on pourrait qualifier d'humaniste. On peut se demander si la présence d'Ippolito d'Este dans cette comparaison est légitime: son statut, ses relations, sa situation matérielle lui auraient permis de se procurer plus de livres que tous les curés du pays pris ensemble. Les livres que nous lui connaissons sont, exclusivement, des ouvrages d'auteurs classiques et d'humanistes comtemporains. Sa collection illustre cette culture tres particulière des prélats de sa génération: on peut observer une composition thématique semblable dans les bibliothèques des archévêques d'Esztergom, ainsi

45 Après les trois éditions hongroises du registre, une édition a vu le jour en Slovakie également (sans renvoyer aux publications hongroises): Helena SAKToRova, Najstarši katalóg knižnice rodu Zaiovcov, Kniha 2016, 335-342.; Michaela SibyLova, Unikát z knižnej zbierky baróm Františku Zaia (1505-1570) $v$ fonde Univerzitnej knižnice v Bratislave, Studia Bibliographica Posoniensia, 12(2017), 34-42. 
que celles des évêques de Vác, Pécs, Eger ou Várad. ${ }^{46}$ Si un Georg Petri, simple curé paroissial de Bártfa, pouvait se prévaloir d'une bibliothèque humaniste, on peut légitimement supposer que la culture de ces confreres appartenant au moyen-clergé pouvait être de nature semblable ${ }^{47}$.

Afin de ne pas abuser de votre patience, je suspends l'analyse du corpus des bibliothèques privées, mais je me permets d'en tirer quelques conclusions. Il est évident que jusqu'aux premières décennies du XVII éècle, l'élite intellectuelle en Hongrie et en Transylvanie n'était nullement en retard par rapport aux courants intellectuels européens. Par contre, la guerre de quinze ans (1591-1606), ainsi que les confrontations confessionnelles de la première moitié du XVII ${ }^{\mathrm{e}}$ siècle ont empêché les érudits de s'acquérir les publications occidentales récentes. ${ }^{48}$ Apres l'expulsion des Ottomans, les efforts de recatholisation forcée (auxquels l'Etat avait assisté) et la marginalisation systématique du réseau institutionnel protestant n'ont fait quaggraver les tendances défavorables. Notons enfin que la cour des Habsbourg, travaillant à la modernisation administrative du pays, n'a pas réussi à imposer l'allemand comme langue officielle: le pays s'est accroché au latin traditionnel. Seule la couche la plus élevée de la société, les aristocrates ont appris le

$46 \quad$ Sur la culture humaniste des prélats actifs sous le règne de Mathias et à l'époque des Jagellons, il existe un tas d'études. Une bonne synthèse se trouve dans le catalogue de l'exposition ayant lieu en 2008: Mátyás király öröksége, Késő reneszánsz müvészet Magyarországon: 16-17. század - King Mathias' Heritage, Late Gothic Art in Hungary: sixteenth and seventeenth centuries, Exhibition in the Hungarian National Gallery, 2008. március 28.-július 27, eds. Árpád Мiкo, Mária Vero, Budapest, MNG, 2008.; vö. Árpád Мiкo, A reneszánsz Magyarországon, Budapest, Corvina, 2009.; Gábor Farkas KIss, Ambiguity and Paradox in the Humanistic Literature of the Jagiellonian Age, = Armed Memory: Agency and Peasant Revolts in Central and Southern Europe (1450-1700). Ed. Gabriella Erdelyi, Göttingen, Vandenhoeck und Ruprecht, 2016, 171-190.

47 Vö. Köвlös József, Az egyházi középréteg Mátyás és a Jagellók korában, Budapest, MTA TTI, 1994 (Társadalom- és művelődéstörténeti tanulmányok, 12.)

48 Katalin Peter, Golden Age and Decay in Intellectuel Culture at the Turn of the Seventeenth Century, = Katalin Peter, Studies on the History of the Reformation in Hungary and Transylvania, ed. by Gabriella ERDelyI, Göttingen, Vandenhoeck und Ruprecht, 2018 (Ref500 Academic studies, 45), 149-178. 
francais, tandis que l'allemand est resté la premiere langue étrangère (il était, bien entendu, la langue maternelle d'une certaine bourgeoisie). Le résultat: l'archaisation progressive des lecture des intellectuels du bassin des Carpathes. Nous connaissons quelques bibliothèques privées du début du XIX ${ }^{\mathrm{e}}$ siècle, dont le corpus se compose, presqu'exclusivement, de livres publiés au XVII siècle. 
LES BIBLIOTHÈQUES ET L'ÉCONOMIE DES CONNAISSANCES Bibliotheken und die ÖKonomie des Wissens

$$
145^{\circ}-185^{\circ}
$$

Colloque international - Internationale Tagung 9-13 avril/April 2019 Sárospatak (Hongrie/Ungarn)

Édité par

Frédéric Barbier, István Monok et Andrea Seidler 


\section{L'Europe en réseaux}

Contribution à l'histoire de la culture écrite 1650-1918

Vernetztes Europa

Beiträge zur Kulturgeschichte des Buchwesens 1650-1918

Édité par

Frédéric Barbier, Marie-Elisabeth Ducreux, Matthias Middell, István Monok, Éva Ringh, Martin Svatoš

Volume VIII

École pratique des hautes études, Paris

École des hautes études en sciences sociales, Paris

Centre des hautes études, Leipzig

Bibliothèque nationale Széchényi, Budapest

Bibliothèque et centre d'information de l'Académie hongroise des sciences, Budapest 
LeS BIBLIOTHÈQUeS ET L'ÉCONOMIE DES CONNAISSANCES BibliotheKen und die ÖKonomie des Wissens

$$
145^{\circ}-185^{\circ}
$$

Colloque international - Internationale Tagung 9-13 avril/April 2019 Sárospatak (Hongrie/Ungarn)

Édité par

Frédéric Barbier, István Monok et Andrea Seidler

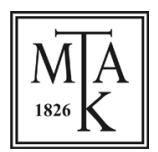

Magyar Tudományos Akadémia Könyvtár és Információs Központ Budapest 2020 
Mise en page

Viktória Vas

ISBN 978-963-7451-57-7

DOI 10.36820/SAROSPATAK.2020 
Préface

István MoNOK

Bibliothecae mutantur - Quare, quemadmodum et quid attinet?

Transformations de la composition thématique

des bibliothèques du Royaume de Hongrie aux $\mathrm{XV}^{\mathrm{E}}-\mathrm{XVI} \mathrm{I}^{\mathrm{e}}$ siècles....11

Marianne CARbonnier-Burkard

Les bibliothèques des Églises réformées françaises au XVII siècle.... 30

Max Engammare

De la bibliothèque de l'Académie de Calvin (1570) a la bibliothèque de l'Académie de Bèze (1612) à travers leur catalogue: Continuités et ruptures jusqu'au troisième catalogue de 1620........... 57

Róbert OLÁ́

Obsolescent Reformed Libraries in the seventeenth and eighteenth Century Carpathian Basin

Ádám Hegri

Moderner Zeitgeist - veraltete Lesestoffe. Bibliotheken reformierten Pfarrer um die Wende des 18. zum

19. Jahrhunderts im Königreich Ungarn

Petr MAšEK

Zierotin Library in Velké Losiny in Sixteenth and Seventeenth century. 136

Detlef HABERLAND

Schlesische Bibliotheken Zeichen der intellektuellen Vielfalt einer zentralen Bildungsregion in Europa 146

Thomas WaLLNIG

Sebastian Tengnagel und Johann Seyfried - Österreichische

Geschichtsschreibung zwischen Späthumanismus und

Gegenreformation 
Elisabeth EngL-Ursula RautenBerg

Christoph Jacob Trew - Bibliothek und Sammeln in der Gelehrtengemeinschaft der ersten Hälfte des 18. Jahrhunderts.

Helwi BLOM

Philosophie ou Commerce?

L'évolution des systèmes de classement bibliographique dans les catalogues de bibliothèques privées publies en France au XVIII ${ }^{\mathrm{e}}$ siècle.

Maria Luisa López-VIDrIero Abelló

Les meubles de la connaissance: façons de devenir sage à prix fixe.

Frédéric BARbier

Distinction, récréation, identité: la trajectoire des « romans» en France sous d'Ancien Régime.

\section{Andrea SeIdler}

Die praktische Bedeutung ungarischer Sammlungen und Bibliotheken für führende Gelehrte des Königreichs Ungarn im späten 18. Jahrhundert am Beispiel des Jesuiten Georg Pray (1723-1801).

\section{Olga Granasztói}

Se divertir: les enseignements de la bibliothèque d'une femme aristocrate hongroise à la fin du XVIII siècle

Christophe Didier

La naissance du théâtre „des boulevards”, ou Comment la banlieue entre en bibliothèque (1780-1830).

\section{Andrea De Pasquale}

La nascita delle riserve di libri antichi in Italia

Index des noms de personne et de lieu..... 